\title{
Délimitation des territoires et rapports de pouvoir dans la zone diamantifère de Séguéla (Côte d'Ivoire) : le lien social familial en question
}

\author{
Bah Mahier Jules-Michel, \\ Niamke Jean Louis, \\ Traore Tiamba,
}

Université Félix Houphouët-Boigny, Abidjan, Côte d'Ivoire

Doi:10.19044/esj.2020.v16n20p142 URL:http://dx.doi.org/10.19044/esj.2020.v16n20p142

\section{Résumé}

L'étude vise à analyser les changements opérés dans les rapports interfamiliaux en lien avec le processus de délimitation des territoires des villages dans la zone diamantifère de Séguéla. L'étude a consisté à recueillir les données qualitatives à partir de la recherche documentaire et l'entretien. Les résultats obtenus indiquent que le processus de délimitation initié durant ces dernières années dans la zone diamantifère de Séguéla, a permis aux grandes familles de revisiter leurs liens ancestraux avec les enjeux socioéconomiques du moment. Cela passe par la mise en place de stratégies de contrôle familial et par la réaffirmation du contrôle foncier à travers le renouvellement de redevabilité. Ainsi, l'alignement ou le désalignement des acteurs locaux aux liens socio-historiques et la dynamique économique dans la région renforcent ou affaiblissent la cohésion sociale entre les familles autochtones dans les villages.

Mots-clés: Zone diamantifère, délimitation des territoires, stratégies familiales, cohésion sociale 


\title{
Delimitation of territories and power relations in the diamondiferous zone of Séguéla (Cote d'Ivoire): the family social link in question
}

\author{
Bah Mahier Jules-Michel, \\ Niamke Jean Louis, \\ Traore Tiamba,
} Université Félix Houphouët-Boigny, Abidjan, Côte d'Ivoire

\begin{abstract}
The study aims to analyze the changes made in interfamilial relations in relation to the process of delimitation of village territories in the Séguéla diamondiferous zone. The study consisted of collecting qualitative data from desk research and interview. The results obtained indicate that the delimitation process initiated in recent years in the diamondiferous zone of Séguéla has allowed large families to revisit their ancestral links with the socio-economic issues of the moment. This requires the implementation of family control strategies and the reaffirmation of land control through the renewal of accountability. Thus, the alignment or misalignment of local actors with sociohistorical links and the economic dynamics in the region reinforce or weaken the social cohesion between the indigenous families in the villages.
\end{abstract}

Keywords: Diamond zone, territorial boundaries, family strategies, social cohesion

\section{Introduction}

L'utilisation paisible et durable de la terre constitue l'un des objectifs affichés de la sécurisation foncière en milieu rural ivoirien. La formalisation des droits d'usages et de propriété à partir de certificat et de titre foncier sont autant de mécanismes étatiques de lutte contre l'insécurité foncière à l'échelle micro. Mais au niveau macro, la délimitation des territoires villageois joue ce même rôle. Ainsi, l'importance d'un tel processus n'est plus à démonter. Il permet aux villages de connaître non seulement leurs limites, mais aussi d'avoir une carte de leur territoire (FAO, 2017).

Cependant, quoique d'actualité en Côte d'Ivoire, les défis de la délimitation des territoires des villages restent peu documentés. Les différentes contributions sur la question foncière portent essentiellement sur la sécurisation foncière, le cadre juridique du foncier rural, l'implémentation 
de la loi foncière de 1998. Ils indiquent que la loi de 1998 a pour but de réglementer le domaine foncier rural suivant des dispositions, des objectifs visant à moderniser l'occupation et la gestion des terres rurales (Chauveau, 2006 ; Zalo, 2006). Cela renforcerait la cohésion sociale à l'intérieur des villages et entre villages voisins en ré-établissant un climat de confiance et de réconciliation entre les communautés rurales, en réduisant les litiges fonciers et en facilitant leurs règlements.

Au regard de la grande portée d'un tel projet, triste est de constaté que ce processus de délimitation dans un contexte du Worodougou laissant la zone diamantifère de Séguéla dans de potentielles crises foncières interfamiliales. Les destructions volontaires de cultures ${ }^{3}$ dans certains villages et le blocage du processus de délimitation ${ }^{4}$ dans d'autres, sont autant d'indices de potentiels conflits dans la région. Cette inquiétante situation met en mal la cohésion sociale dans la localité.

Cette recherche qui a pour objectif principal d'analyser les changements opérés dans les rapports interfamiliaux en lien avec le processus de délimitation des territoires des villages dans la zone diamantifère de Séguéla vise de manière spécifique à : i) cerner les stratégies familiales de contrôle des terres initiées par les familles autochtones autour de la délimitation des territoires ; et ii) étudier le renouvellement de la redevabilité qu'elles induisent.

\section{Méthodologie}

La méthodologie mise en œuvre a consisté à conduire des enquêtes de terrain dans les Sous-préfectures de Séguéla, Dualla, Diarabana et Bobi. Le choix de ces Sous-préfectures dans la réalisation de cette étude est motivé par le processus de délimitation des terroirs à l'œuvre dans cette zone depuis 2014. Ainsi, le choix des villages dans ces localités s'est fait à partir de la diversité d'expression du processus de délimitation dans ces zones.

A ce jour, le processus de délimitation appuyé par le projet Droits de Propriété et Développement du Diamant Artisanal II (DPDDA II) permet de classer les villages impliqués en 3 catégories. Il s'agit des villages totalement délimités à savoir Massala-Assolo, Sangana, Dualla, Oussougoula, Niéou, Sokoura, Dafana et Dona; des villages partiellement délimités à savoir Niongonon, Diarabana, Forona et Bobi ; et des villages qui ne sont pas pris en compte dans le processus de délimitation, mais qui y ont été impliqués dans la mesure où ils font frontière avec certains villages directement concernés à

\footnotetext{
${ }^{3}$ En 2017, il y a eu des destructions des plants d'anacarde dans deux villages à savoir DonaSomana et Dona-Siana. Ces acteurs estimaient que ces champs se trouvaient dans leur patrimoine foncier. Cette même opération s'est également déroulée dans deux autres villages à savoir Dona-Siana et Niongonon-Diarabana en Mars et Juin 2018.

${ }^{4}$ Tronçon de limite Niongonon-Diarababa et celui de Forona-Bobi.
} 
savoir Siana, Suinla, Mamouroula, Somana et Wongué. De ce fait, 12 villages dont 4 villages par catégories présentés ont été choisis à partir de la technique d'échantillonnage de milieu. Il s'agit de Dona, Oussougoula, Sangana, Dualla, Forona, Diarabana, Niongonon, Bobi, Somana, Siana, Wongué et Suinla. Cela a permis la triangulation des points de vue des acteurs familiaux interviewés. Le choix de ces acteurs familiaux s'est fait prioritairement en fonction de leur implication dans le processus de délimitation. Il s'agit des personnes appartenant à la notabilité, au comité villageois de gestion de foncier rural (CVGFR) et des leaders communautaires (chefs des communautés).

La collecte des données s'est faite durant la période allant de mai 2017 à juillet 2018. Il faut noter qu'elle ne s'est pas faite de façon linéaire. La technique utilisée a été l'entretien individuel. Elle a été menée auprès de 23 acteurs familiaux entre autres 5 chefs du village, 4 Secrétaires de la notabilité, 6 responsables des jeunes, 7 Secrétaires de CVGFR et 1 agent local de la SODEMI selon la technique d'échantillonnage par choix raisonné.

Pour traiter les données recueillies, nous avons eu recours à l'analyse de contenu qui est l'ensemble des analyses de texte ou d'énonciation élaborées pour traiter des réponses non immédiatement quantifiables (Durand \& Weil, 2006). La procédure comprend généralement la transformation d'un discours oral en texte, puis la construction d'un instrument d'analyse pour étudier la signification des propos (Bardin, 1977). Il convient, en effet, de rassembler des informations parfois ambigües, incomplètes et contradictoires, d'interpréter les similitudes et les différences entre les répondants et de parvenir à une analyse objective (Andreani \& Conchon, 2005).

De façon pratique, le traitement des données qualitatives a consisté à faire un inventaire, puis un tri des informations obtenues à partir des observations et des entretiens en vue de procéder à des classifications et à des recherches de correspondances entre les variables sélectionnées. Ce travail préparatoire a permis de mettre en relation les données sélectionnées et leur contexte de production. En effet, il s'est agi de privilégier l'écoute plutôt que le questionnement, la compréhension et l'analyse en profondeur plutôt que le dénombrement. Ainsi, l'analyse de contenu a suivi trois (3) principales caractéristiques à savoir la retranscription des données contenues dans les différents guides d'entretien, le codage des données, et le traitement des données.

\section{Résultats}

La carte ci-dessous présente les différents villages diamantifères de Séguéla. 


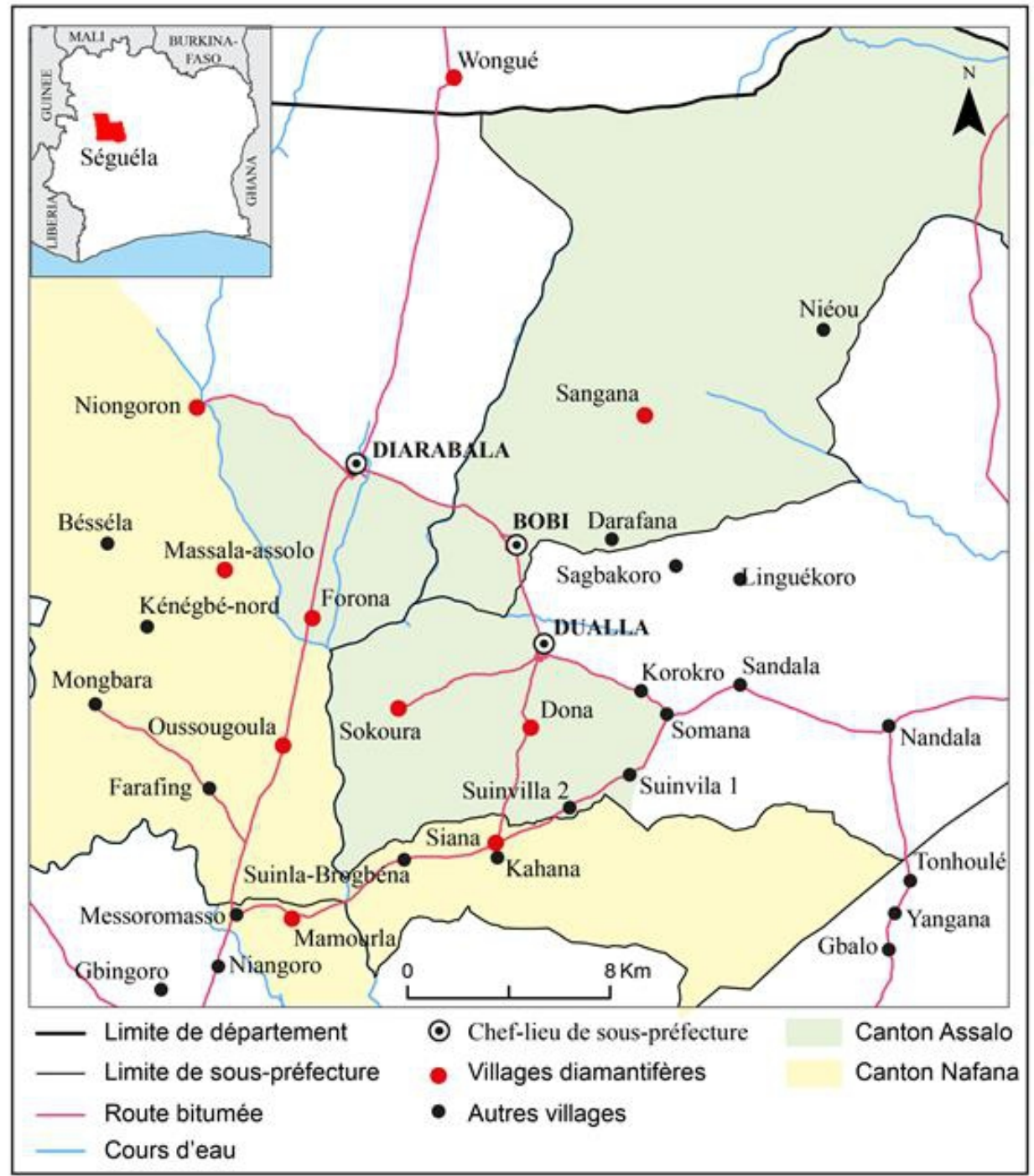

Source: CNTIG, 2014; Enquêtes Bah, 2019

Réalisation: Kouadio, 2019

Deux principaux résultats ont été obtenus. Ce sont :

1) la délimitation des territoires comme stratégie familiale de contrôle des terres ;

2) la délimitation des territoires comme renouvellement de redevabilité.

\section{2-1. La délimitation des territoires comme stratégie familiale de contrôle des terres}

Les discours sur la délimitation des territoires établissent diverses stratégies familiales de contrôle ou de réaffirmation du contrôle sur les terres des villages perçues comme bien communautaires c'est-à-dire appartenant à l'ensemble des familles autochtones fondateurs du village, ou familiales c'està-dire appartenant à des familles spécifiques du village. Ces stratégies familiales font usage des relations socio-historiques entre les familles 
créatrices des villages et les migrants. Elles sont principalement tournées vers la redéfinition des rapports fonciers interfamiliaux, la revendication des terres familiales, le désir de confirmation de la propriété des espaces de délimitation fait par la Société pour le Développement des Mines (SODEMI).

\section{2-1-1. La redéfinition des rapports fonciers interfamiliaux}

Le processus de délimitation des territoires se positionne comme un outil de redéfinition des rapports fonciers à interfamiliaux à l'intérieur des villages et entre des villages voisins. Certaines restrictions consensuelles de l'usage des terres pourraient être mises à l'actif de la connaissance des limites des terres du village. En effet, la tracée des limites du territoire villageois est souvent vu par les grandes familles propriétaires terriens comme une occasion de défense ou de protection voire de redéfinition de l'usage des terres du village. La redéfinition du mode d'occupation de l'espace se présente comme un mécanisme local de contrôle ou de réaffirmation du contrôle sur les terres familiales. Cela se passe également par des consensus, autour des tronçons de limites et de l'usage des potentielles ressources naturelles génératrices de revenus entre les grandes familles propriétaires terriens pour le compte de leur village respectif. Le consensus autour de l'usage des potentielles ressources naturelles génératrices de richesse comme la colline Mankongnon ${ }^{5}$ en est un exemple. Il y a aussi des interdictions ou limitations de pratiquer ${ }^{6}$ les cultures de rente, la définition des espaces réservés aux cultures vivrières ou aux réserves foncières sont des exemples de limitation et de redéfinition de l'occupation des espaces.

Le récit de $F . S$., Secrétaire de la notabilité de Diarabana indique ce fait : «Certains essaient encore d'agrandir leur champ. Le chef a dit d'arrêter ; pas pour nous, mais pour nos enfants...».

Cette mesure prise à l'égard des autochtones peut être mise à l'actif de leur prise de conscience de l'amenuisement des terres culturales révélées par le processus de délimitation et les diagnostics réalisés par le projet DPDDA II. En effet, la délimitation des terres et les différents diagnostics menés dans la zone diamantifère de Séguéla ont révélé l'amenuisement des espaces culturales due à l'émergence de la culture de l'anacarde dans toutes les localités et celle du cacao dans certains cas (DPDDA II, 2018, 2017).

${ }^{5}$ La colline Mankongnon a été l'objet d'un consensus entre les villages de Sangana et de Wongué. Après de nombreux désaccords sur les limites, la notabilité de ces deux villages en présence de celles du village Somana avec pour témoin les Sous-préfets de Kani et de Bobi, ont décidé que les ressources générées par cette colline dans le futur soient partagées entre eux.

${ }^{6}$ Pendant que certains villages ont résolument interdit aux non autochtones la pratique des cultures de rentes, d'autres par contre ont interdit l'agrandissement des champs. D'autres villages ont également réservé des zones spécifiques comme la savane aux autochtones originaires du village. 


\section{2-1-2. La revendication de la terre familiale}

La délimitation des territoires a souvent entraîné une occasion de revendication de la terre familiale. Il y a eu souvent une confusion entre la délimitation du villageois et le partage de la terre familiale comme l'évoque F. V., Secrétaire générale de la notabilité de Forona à travers les propos suivants :

«Tu vois, c'est notre parent qui est installé à Bobi qui complique tout. On parle de délimitation des villages, mais il veut qu'on partage la terre des Fofana afin qu'il puisse mettre sa part sur le territoire des Koné. C'est là le problème...».

En effet, il y a des villages qui partagent une même frontière. Un autochtone d'un village A peut être installé dans un village B. La terre familiale de ce dernier peut être à la lisière de son village hôte. Il peut alors souhaiter que sa portion de terre fasse partie de son village d'origine ou le contraire. Mais, la flexibilité ou la rigidité des autres membres de la famille peut être un facilitateur ou un frein à la tracée de tronçon de limite.

\section{2-1-3. Le désir de confirmation de la propriété des espaces de délimitation}

Certaines logiques de délimitation se fondent sur le désir de confirmation de la propriété de parcelle de diamant. En effet, Koffi (2008) indique que l'exploration du sous-sol ivoirien a dans un premier temps nécessité l'expertise privée comme la Société Diamantifère de Côte d'Ivoire (SODIAMCI). Cependant, soucieux de promouvoir l'industrie minière, l'Etat ivoirien a initié une politique de mise en valeur du secteur minier particulièrement celui du diamant. Cette politique volontariste est marquée par l'adoption d'un code minier résultant de la loi nº64-249 du 3 juillet 1964 et des textes pour son application et la création de la Société pour le Développement des Mines (SODEMI). Aussi, a-t-elle privilégié la mise en place d'un plan de développement progressif du secteur minier en deux phases axées sur la pacification de la filière et le contrôle de la chaîne de production aidant l'exploration et l'inventaire des ressources minérales et permettant une exploitation rationnelle du secteur. Dans la chaîne d'exploitation du diamant, c'est cette structure qui est habilitée à délimiter les parcelles pour une potentielle exploitation artisanale. Mais, les discours des acteurs locaux sur la tracée de tronçons de limites font mention d'une confusion entre délimitation des territoires des villages et délimitation SODEMI. En effet, la délimitation faite par la SODEMI sur des sites propices pour l'exploitation artisanale du diamant depuis 1986 est considérée comme un indice de propriété de terre par certains acteurs. Le discours de F. L., Membre du CVGFR de Forona corrobore cette idée. Il s'exprime en ces termes : 
«On a donné le maximum d'information. Maintenant, on a constaté qu'on ne marchait plus sur la vérité. Chacun défendait son intérêt personnel. Quand c'est comme ça, ça devient compliqué. On a vu même l'exemple de Diarabana-Bobi, de Diarabana-Niongonon. Il restait maintenant à défendre l'intérêt personnel puisque, avant l'arrivée des blancs, c'est Diarabana qui exploitait le site appelé SODIAMCI. Les blancs, avant de s'installer pour l'exploitation de diamant, ce sont Fofana de Diarabana qu'ils ont vu...».

Ces propos montrent bien l'enjeu du positionnement de chaque partie. L'économie du diamant participe au refoulement des limites traditionnelles pour la délimitation SODEMI. Alors que la délimitation SODEMI ne prenait pas en compte la propriété terrienne.

Encadré 1: Propos de K. S, Agent de la SODEMI à Bobi

Je vais partir toujours de l'historique. On les a organisés en groupement, en GVC. On leur dit que ce qui est important, c'est le développement de leur village. Ne dites pas non, c'est ma terre ici, je ne donne pas aux autres. Permettez que les autres villages viennent travailler chez vous aussi. Il y a eu cette libéralisation-là qui fait qu'il y a des villages qui sont allés demander des terrains à d'autres villages. Je prends par exemple le cas de Kani où le permis n'arrivait pas. Notre permis s'arrêtait à Wongué. Donc, ils sont venus demander des terrains aux gens de Wongué. Il y avait deux GVC à Kani. Ils sont venus demander des terrains aux gens de Wongué. C'était faisable à l'époque. Et donc, il n'y avait pas trop ce problème de délimitation ! Ils venaient, on délimitait cent hectares. Ils nous disent qu'il y a un marigot qui est là. Nous, on vient, on délimite ! On demande simplement à qui appartient la zone. Ils nous disent que c'est pour nous ou bien nous sommes allés demander dans tel village. Ils nous ont fait une lettre pour dire qu'ils nous ont accordé la zone. Et, nous on leur cédait la zone. Nous, on partait juste délimité. Une fois qu'on a fait notre enquête pour voir si la zone n'est pas litigieuse, on va délimiter. C'était comme ça qu'on faisait à l'époque. Nous, on ne tenait pas compte du fait que ce n'est pas dans leur village. La preuve en a qu'il y a des gens de Séguéla qui ont eu des parcelles à Suinla!

Cette libéralisation de parcelle aux autres villages est postérieure à la crise socio-politique de 2002. Mais après cette crise, on assiste à une polarisation du marquage des parcelles de diamant. La propriété des lopins de terre contenant une parcelle est alors revendiquée. Cette revendication tient souvent compte de la propriété de parcelle ou la propriété des pères. Cette polarisation des revendications de la propriété lors du processus de délimitation serait une opération portée par des jeunes autochtones dits éclairés. 
Encadré 2 : Propos de K. S, Agent de la SODEMI à Bobi

C'est après la crise que les choses ont commencées à se mélanger ici. Avant, il n'y avait pas de problème. Il n'y avait pas de problème foncier. C'est ce qui s'est passé à Mamourla. A Mamourla, il y a une rivière qui sépare les deux villages (Mamourla/Suinla) et les limites étaient avec les rivières d'ailleurs. Et quand on devrait donner une zone aux gens de l'Entente'. [...]. Sinon à l'époque, il n'y avait pas de problème. Voilà comment on délimitait une zone. Les gens viennent. Ils nous font une demande par écrit. Une fois qu'ils ont fait la demande, nous, on regarde. On fait une enquête pour voir si la zone n'est pas objet de conflit. Si c'est dans un village, on se rend, on discute. Si on voit qu'il n'y a pas de problème, on s'en va et on délimite la zone avec les villageois eux-mêmes. Léonard Grogoué même est venu faire un film ici à l'époque. Quant il est parti, on lui est donné un terrain à Diarabana où il a exploité. Léonard Grogoué. Sa parcelle s'appelait Ivoire Or. Il n'y avait pas de problème !

Comme le montre bien ce témoignage l'activisme de certains acteurs locaux, participe à maintenir la confusion entre la délimitation des terroirs villageois et celle des parcelles d'exploitation de diamant. Néanmoins, le recours à la carte de délimitation des parcelles et au ressassement du processus d'attribution de ces parcelles peuvent éclairer le processus de levée de blocus sur les espaces convoités.

\section{2-1-4. La renégociation des territoires villageois}

Le processus de délimitation est aussi vu comme une opportunité d'agrandissement du terroir villageois. Les stratégies de positionnement des acteurs familiaux montrent bien cette volonté d'acquérir de nouveaux espaces et de formaliser sa possession. C'est le cas de Dualla et de Sokoura. Les autochtones de ces villages ne seraient pas propriétaires terriens. Ils seraient redevables à au Koné, Fofana, Diomandé et Dosso. C'est pourquoi durant le processus de délimitation, ces villages auraient joué la carte de l'apaisement. Ils n'ont pas discuté la propriété d'un espace quelconque, mais ont-ils procédé par demande et reconnaissance de la propriété des villages à la lisère des siens. Avec des démarches initiées par la chefferie des ces villages avec l'appui des leurs alliés Coulibaly de Niongonon pour les Soumahoro de Dualla et Dosso pour les Coulibaly de Sokoura, ces villages ont fait la délimitation de leur village sans conflits majeurs.

\footnotetext{
${ }^{7}$ C'est un groupement à Vocation Coopérative basé à Séguéla. Il est composé essentiellement d'allochtones.
} 
Encadré 3 : Propos de C. I., Secrétaire général de la notabilité de Sokoura

Il y a Oussougoula, il y a Suinla, il y a Forona, il y a Dona. C'est avec ces villages que nous avions tracé nos limites. Pour les accords de limites, tu sais, dans la vie, tout le monde n'est pas né le même jour. Tout le monde ne peut pas mourir le même jour. Les Tchomba qui sont passés, chacun connaît comment il s'est installé. De la manière les Tchomba se sont montrés les coins, chacun connaît se qui l'appartient. Même si le Tchomba n'est plus de ce monde, il a transmis sa connaissance du milieu à sa descendance. Il a montré aussi les frontières du village avec les villages voisins. Il a aussi laissé l'histoire de l'installation du village et celui de ces voisins. Mais, à cause de la course au diamant, chacun certains devient avide d'espaces. Et, certains espaces sont revendiqués malgré que celui qui le revendique soit conscient qu'il n'est pas à lui.

Cette forme de revendication basée sur le déni de la propriété de l'autre met souvent en mal la concorde entre familles autochtones comme le dénote $C$. $S$, le président des jeunes de Sokoura:

"A cause de la course au diamant, certaines familles forcent l'appartenance des terres. C'est ce qui emmène beaucoup de conflits. Si tu n'as pas envie de mentir, comme tu connais comment tu t'es installé, comme les Tchomba t'ont montré les limites, avant la délimitation, les villages doivent s'entendre».

Le dialogue entre les différentes familles a été un apport certain pour la facilitation de la tracée des limites. Cependant, ce dialogue s'inscrit dans le jeu local de la redevabilité.

\section{2-2. La délimitation des territoires comme renouvellement de la redevabilité}

L'étude montre que les accords de limite entre les villages s'opèrent dans un jeu local de redevabilité. Les grandes familles propriétaires terriens exigent que leur autorité soit d'abord reconnue avant toute négociation ou accords de limites. Ce jeu local de la redevabilité est mis à l'actif de la tracée paisible des limites des territoires villageois.

\section{2-2-1. La redevabilité comme stratégie consensuelle de tracé paisible de limites}

La tracée paisible des tronçons de limites est mis à l'actif de l'alignement des grandes familles autochtones au jeu local de la redevabilité. 
Encadré 4: Propos de C. I, Secrétaire général de la notabilité de Sokoura

Si tu dois demander à ce qu'une portion de terre fasse partir de ton territoire, ce n'est pas avec la force ! Il faut reconnaître d'abord ta limite historique. Après tu pourras demander à ce que cette limite soit revue en ta faveur. Chez nous, il n'y a pas eu d'histoires. [...].Au cours de la délimitation, nous sommes partis, comme chacun connaît sa limite, personne n'était avide d'espace. Oussougoula et nous avions parlé avec la même voix. C'est comme ça que pour nous s'est passé. Arrivé à Suinla, même chose. Avec Dona, même chose. Pour Dona, il y avait un coin, nous savons tous que c'est la terre de Dona. Nous avons reconnu qu'ils sont les propriétaires. Nous n'avons pas fait d'histoire, nous les avons suppliés de nous laisser cette partie de leur territoire. Nos Tchomba se sont levés pour aller voir les Tchomba de Dona pour ça. Tellement ils étaient contents, ils nous ont laissé une part au-delà de ce qu'on avait demandé. Et on a fait la délimitation. C'est comme ça! Ils ont ajouté d'autres parts sur la part de nos Tchomba. Comme ça, avant la délimitation, nous nous sommes levés pour rentrer tous nos villages voisins pour le respect et la fraternité. Par ce que c'est dans le respect qu'on peut tout avoir. Les Tchomba ont fait une délégation pour aller à Dona, une délégation pour aller à Forona et une délégation pour Oussougoula. C'était pour qu'on puisse s'entendre avant la délimitation.

Ce discours révèle bien l'alignement au jeu local de la redevabilité comme instrument au service de la tracée des limites des territoires villageois dans la quiétude. Les grandes familles se sont alignées aux formes locales de reconnaissance de propriété foncière dans la plupart des cas pour sauvegarder la bonne entente.

Encadré 5 : Propos de D. M, Président des jeunes et secrétaire de la notabilité de MassalaAssolo

J'ai eu cette chance. Certes, je n'ai pas trop connu mon père qui décédé depuis 1984, mais j'ai eu un grand-père, qui était l'un des grands sachants du canton Assolo-Nafana pour ne pas dire toute la région. Et, ce vieux, à vraiment eu confiance en moi et m'a confié, des secrets de savoir en ce qui concerne les différents territoires. Par exemple Oussougoula que vous voyez, d'après le vieux, le territoire qui vient vers Massala-Assolo, appartenait à des Dosso de Oussougoula qui l'ont attribué à des Bakayoko. [...] Ce même territoire de Massala-Assolo qui va vers Oussougoula, appartenait à la famille Dougoula de MassalaAssolo. Le vieux de cette famille a confié cette portion de terre à des Koné. [...]. Donc, c'est deux étrangers qui se font frontière. [...]. Mais qu'est-ce qu'il faut faire ? En cas de délimitation, il faut appeler les deux sources qui sont les Dosso du côté de Oussougoula et les Dosso du côté de Massala-Assolo. C'est ceux-là qui doivent vraiment s'entendre pour faire la tracée des layons. Ils doivent discuter. [...].Quand c'est arrivé, la famille Dosso de Massala-Assolo s'est rendue à Oussougoula. Nous avons demandé la paternité de l'espace qui se trouve du côté de notre village. Le vieux de la famille Dosso de Oussougoula a dit que ce tronçon appartient à des Bakayoko. Nous avons dit alors que les Bakayoko n'ont pas de terrain ici. [...]. Les Bakayoko n'ont pas de terre dans le canton ici, la terre a été attribuée aux Bakayoko par des Dosso. [...]. Les Bakayoko ne peuvent pas nous montrer les limites de leurs terres. Ils n'ont pas de terre ici ! Les Koné de chez nous ont dit qu'ils ont des terres qui leur ont été confié. Qu'ils n'ont pas de limites à montrer puisque les vrais propriétaires sont les Dosso et que c'est cette famille qui peut montrer la limite et qu'ils les suivent. Et, les deux familles Dosso de Massala-Assolo et Oussougoula se sont entendues et on n'a fait la tracée du layon. 
Ce discours retrace les mécanismes locaux d'accords autour des limites des territoires villageois dans la zone diamantifère de Séguéla. En effet il y a une différenciation entre les autochtones propriétaires terriens dans les différents villages. Ils sont liés par le jeu de la redevabilité qui interfère dans les interactions de tracée de limites des territoires villageois. Le déni de redevabilité est alors perçu par les acteurs familiaux comme sources.

\section{2-2-2. Le déni de redevabilité comme source de tensions interfamiliales}

La plupart des interactions entre les grandes familles pour la tracée de limite, s'opèrent dans un contexte de rappel des liens historiques qui lient les grandes familles. Toutefois, le déni de redevabilité est perçu comme source de tension et de dissension sociale. Cela expliquerait les différents désaccords autour des tronçons de limites notamment entre Niongonon-Diarabana et Forona-Bobi.

Encadré 6: Propos de D. M, Président des jeunes de Massala-Assolo et secrétaire de la notabilité

D'après ce que nous avons appris de nos grands parents, Niongonon est peut-être à son troisième site où ils sont actuellement. [...]. Niongonon était derrière le fleuve kohoué. Je peux même dire, un peu sur le territoire de Wongué. [...]. Niongonon, étant à Sodjamissi. Ce village faisait frontière avec Wongué. [...]. Vous savez que, à l'époque, s'était la loi du plus fort. [...]. C'est ainsi que les parents sont venus installer Niongonon. [...]. Sinon, leur premier village se trouve de l'autre côté du fleuve en allant vers Wongué qui est aujourd'hui le site de Sodiamissi. Le nerf du problème entre Niongonon et Diarabana, est qu'il y a eu un site que les blancs exploitaient. Après leur contrat, ils ont cherché à rentrer. Niongonon, ayant leur premier village sur le site, les blancs ont fait appel aux gens de Niongonon d'aller s'installer à Sodiamci pour refonder leur village. Ils ne se sont pas entendus entre eux. Ça fait que le site est resté vraiment inhabité. Ce même site a été exploité par les gens de Diarabana. [...]. Aujourd'hui, Diarabana se dit que c'est pour eux et Niongonon se dit que c'est pour eux. [...]. Mais, il y a des villages Assolo qui se trouvent de l'autre côté du fleuve. Mais, sur notre côté la limite Assolo c'est le kohoué parce qu'il y a une alliance entre le Assolo et le Nafana. [...]. Mais, aux jours d'aujourd'hui, le sous-sol qu'ils ont découverts au niveau de ce site, de Sodiamci, la profondeur même du sol, c'est-à-dire le Kimberlique, attire l'intention de tout le monde. Or le problème est que, depuis le temps des arrangements, Diarabana occupait la plus grande partie de la zone. Diarabana occupait la plus grande partie et il faisait limite avec Wongué.

Ce discours dévoile les enjeux économiques de la délimitation dans la conception des grandes familles propriétaires terriens. En effet, tout espace concédé est considéré comme une perte en cas d'exploitation à long terme des territoires délimités. 
Encadré 7: Propos de K. S, Agent de la SODEMI à Bobi

Aujourd'hui, comme je le dis, c'est après la crise que les nouveaux éclairés sont venues réclamer leur terre. Comme eux-mêmes, ils ont déjà tous bafouillés, tout est déjà gâté, il faut qu'eux-aussi, chacun maintenant protège son patrimoine. Donc du coup, ils ont commencé à réclamer, «ici, c'est pour nous, ici, c'est pour nous ». Le problème est qu'il ya eu des vieux qui sont partir (décédés). Ils y a aussi des vieux qui sont là, mais qui sont de mauvaise foi, qui veulent pas dire la vérité ou bien ils sont obligés de se taire. Tu vois, quand tu vas à Bobi comme ça, il y a un Fofana qui est à Bobi. Mais, le Fofana de Bobi, lui, il est allé s'installer à Bobi. A cause de lui, Bobi a eu des terres. Lui, il cédait ces là à Bobi puisqu'il est allé s'installé à Bobi. Ces terres là, aujourd'hui, Bobi réclame ça comme étant leur terre. A l'époque, nous on savait qu'il (Bobi) demandait la permission à Lama Fofana. Nous, on était là. Tu vas leur dire ça, ils (les gens de Bobi) vont nier. Ils vont dire qu'ils n'ont jamais fait ça. Les Fofana ne viennent pas de Bobi. Les Fofana, viennent de Diarabana, de Forona puisque c'est la même famille, Diarabana/Forona. Eux, c'est la même famille. C'est eux qui ont cédé une partie de leur terre aux gens de Bobi. Le Fofana qui est là-bas n'arrive pas à dire la vérité.

Cette même lutte pour le contrôle spatial s'oriente également vers les ressources naturelles comme les collines. Le discours de F. A, Secrétaire général de CVGFR, de Diarabana, révèle ce fait :

«Avant délimitation là, les chinois sont venus détecter une carrière chez nous ici. Ils disent qu'ils font faire une usine là-bas. Mais, quand nous on a dit que c'est pour nous, ils nous ont laissé. Ils ont demandé tous les autres villages! [...]. Donc tout ça là, aujourd'hui, parce qu'on a dit qu'il y a carrière, il y a des gens qui sont quittés en Europe, aux États-Unis pour dire qu'ils vont venir enlever Kimberlite à Toubaboukô, il y a des villages qui disputent ces portions de terre avec nous ».

Ce discours dénote du déni de redevabilité due aux enjeux économiques de la localité. En effet, la lutte pour le contrôle des espaces passe par des récits d'occupation qui devraient s'aligner au jeu local de la redevabilité. Mais, ces récits portent souvent à confusion sur la paternité de certains espaces. Cela peut être mis à l'actif de l'oralité de la plus part des transactions foncières (PDDAII, 2018).

\section{Discussion}

Toutes les sociétés s'inscrivent dans une démarche commune qui évolue par phases basées sur l'opposition tradition/modernité (G. Ferréol, 1994). En ce sens la problématique du changement est appréhendée de multiples manières. Certains auteurs font appel à des facteurs endogènes ou exogènes; d'autres, s'interrogeant sur les formes ou les processus qui mettent l'accent sur les conflits ou les effets d'agrégation et privilégient une perspective cyclique ou multilinéaire, faite de ruptures ou de continuités. $\mathrm{R}$. Bourdon (1984) conçoit le changement comme l'aboutissement d'actions cumulées de différents acteurs sociaux. Les mutations sociales s'inscrivent alors dans une perspective au sein de laquelle chaque élément contribue au 
maintien de l'ensemble (G. Ferréol, 1994). Pour K. Marx (1965) le conflit constitue le principal moteur du changement social. Pour lui, toute société se caractérise par son mode et ses rapports de production et toutes les transformations sociales ne se réalisent que par la révolte de la classe dominée contre la classe dominante. Ainsi, de l'exploitation ou de la subordination, dérive la lutte des classes qui est pour lui le moteur du changement social.

Dans le contexte de la zone diamantifère de Séguéla, la crise du lien social a plusieurs visages. Elle est à la fois économique et symbolique. En effet, la pression foncière accentue la valeur économique de la terre entrainant des mutations dans le mode d'accès et d'usage. Le travail partagé et la limitation de l'usage des terres suivant les rapports fonciers interfamiliaux en sont des exemples (DPDDA II, 2018). Ainsi, les crises et les conflits feraient partie intégrante du fonctionnement sociétal des communautés résidentes des zones diamantifères de Séguéla bien avant le processus de délimitation ; il y aurait des conflits latents avant le démarrage du processus de délimitation (DPDDA II, 2014). Mais, le défi a toujours été la capacité des acteurs à les rendre constructifs et pacifiques pour la cohésion sociale. En cela, cette réflexion contribue ainsi à une mise en lumière des stratégies locales de pacification voire d'intensification des conflits fonciers interfamiliaux dans le contexte de la délimitation des territoires villageois.

\section{Conclusion}

La présente étude s'est fixée pour objectif d'analyser les défis autour du processus de délimitation dans les zones diamantifères du Worodougou et les dynamiques qu'ils impliquent dans les rapports entre les différentes familles autochtones originaires des villages. L'approche interactionniste utilisée permet de montrer à partir du discours des acteurs familiaux et de la recherche documentaire, que le processus de délimitation participe à la révision des liens interfamiliaux à travers la construction d'idéologies conservatrice et de redéfinition de la propriété des terres. Cela passe par mise en scène de stratégies familiales contrôle ou de ré-affirmation de contrôle des terres interfamiliaux à travers le renouvellement versus refoulement de la redevabilité. Ainsi, l'alignement ou le désalignement des acteurs familiaux aux liens socio-historiques associés aux dynamiques économiques dans la région fragilise ou renforce la cohésion sociale entre les familles autochtones originaires des villages.

\section{References :}

1. Andreani, J.-C. \& Conchon, F. (2005), Méthodes d'analyse et d'interprétation des études qualitatives : état de l'art en marketing, ESCP-EAP, Paris, INSEMMA. 
2. Balandier, G. (1963)., "Sociologie dynamique et histoire à partir de faits africains". In: Cahiers internationaux de sociologie, vol. 34, janvier-juin, pp. 3-11.

http://classiques.uqac.ca/contemporains/balandier_georges/socio_dyn amique/socio_dynamique_texte.html.

3. Bardin, L. (1998), L'analyse de contenu, 9èmeEdition, Paris, PUF.

4. Boh, C. (2018), Côte d'Ivoire: Raréfaction progressive du diamant à Séguéla, les autorités minières tirent la sonnette d'alarme http://koaci.com/cote-divoire-rarefaction-progressive-diamantseguela-autorites-minieres-sonnent-sonnette-dalarme-115021.html.

5. Boudon, R. (1984), La Place du désordre. Critique des théories $d u$ changement social, Paris, PUF.

6. Chauveau, J-P. (2006), "La réforme foncière de 1998 en Côte d'Ivoire à la lumière de l'histoire des dispositifs de sécurisation des droits coutumiers. Une économie politique de la question des transferts de droits entre autochtones et « étrangers » en Côte d'Ivoire forestière". Montpellier Communication, Colloque international "Les frontières de la question foncière".

http://www.documentation.ird.fr/hor/fdi:010045143

7. DPDDA II. (2014a), Diagnostic terre-conflits. Localité : Forona. Rapport Tétra Tech, USAID-Union Européenne, Mars.

8. DPDDA II. (2014b), Diagnostic terre-conflits. Localité : MassalaAssolo. Rapport Tétra Tech, USAID-Union Européenne, Mars.

9. DPDDA II. (2014c), Diagnostic terre-conflits. Localité : Dualla. Rapport Tétra Tech, USAID-Union Européenne, Mars.

10. DPDDA II. (2014d), Diagnostic terre-conflits. Localité : Oussougoula. Rapport Tétra Tech, USAID-Union Européenne, Mars.

11. DPDDA II. (2014e), Diagnostic terre-conflits. Localité : Bobi. Rapport Tétra Tech, USAID-Union Européenne, Mars.

12. DPDDA II. (2014f), Diagnostic terre-conflits. Localité : Diarabana. Rapport Tétra Tech, USAID-Union Européenne, Mars.

13. DPDDA II. (2014g), Diagnostic terre-conflits. Localité : Dona. Rapport Tétra Tech, USAID-Union Européenne, Mars.

14. DPDDA II. (2014h), Diagnostic terre-conflits. Localité : Niongonon. Rapport Tétra Tech, USAID-Union Européenne, Mars.

15. DPDDA II. (2014i), Diagnostic terre-conflits. Localité : Sangana. Rapport Tétra Tech, USAID-Union Européenne, Mars.

16. DPDDA II. (2014j), Diagnostic terre-conflits. Localité : Sokoura. Rapport Tétra Tech, USAID-Union Européenne, Mars.

17. DPDDA II. (2017a), Diagnostic participatif et perspectives de Développement durable dans les villages diamantifères de la région 
du Worodougou. Rapport Tétra Tech, USAID-Union Européenne, Juillet.

18. DPDDA II. (2017b), Résultats de l'enquête connaissances attitudes pratiques (CAP) auprès des artisans miniers de Séguéla en Côte d'Ivoire. Rapport Tétra Tech, USAID-Union Européenne, Juillet.

19. DPDDA II. (2017c), Synthèse du diagnostic écologique et socioéconomique des forêts galeries des sites diamantifères de Séguéla. Rapport Tétra Tech, USAID-Union Européenne, Juillet.

20. DPDDA II. (2018a), Plan Stratégique Simplifié de développement de Sokoura. Rapport Tétra Tech, USAID-Union Européenne, Juillet.

21. DPDDA II. (2018b), Plan Stratégique Simplifié de développement de Oussougoula. Rapport Tétra Tech, USAID-Union Européenne, Juillet.

22. DPDDA II. (2018c), Plan Stratégique Simplifié de développement de Forona. Rapport Tétra Tech, USAID-Union Européenne, Juillet.

23. DPDDA II. (2018d), Plan Stratégique Simplifié de développement de Diarabana. Rapport Tétra Tech, USAID-Union Européenne, Juillet.

24. DPDDA II. (2018e), Plan Stratégique Simplifié de développement de Sangana. Rapport Tétra Tech, USAID-Union Européenne, Juillet.

25. Durand, J.-P. \& Weil, R. (2006), Sociologie contemporaine, 3ème Edition revue et augmentée, Paris, Vigot.

26. FAO (2017), « Réussir la sécurisation foncière en Côte d'Ivoire », www.fao.org.

27. Ferréol, G. (1994), Histoire de la pensée sociologique. Les grands classiques, Paris, Armand Colin.

28. Ferréol, G. \& Deubel, P. (1993), Méthodologie des sciences sociales, Paris, Armand Colin.

29. Koffi, I. (2008), Les sociétés d'Etat dans le processus de développement de la Côte d'Ivoire de 1960 à 2000: cas de la SODEMI, Mémoire de Maîtrise en Histoire Moderne et Contemporaine, Université d'Abidjan-Cocody,

30. Louise, T. (2002), «La solidarité sociale et les liens intergénérationnels », Notes de recherche. Nouvelles pratiques sociales, 15(1), pp 200-211,

31. Marx, K, (1867), Un chapitre inédit du Capital, http://classiques.uqac.ca/classiques/Marx_karl/capital_chapitre_ined it/capital_chapitre_VI_inedit.pdf.

32. Zalo, L. D. (2006)., Présentation de l'avancement actuel de la mise en auvre de la loi ivoirienne relative au domaine foncier rural, Montpellier, Communication, Colloque international, «Les frontières de la question foncière », https://www.mpl.ird.fr/colloque_foncier/Communications/PDF/Zalo. pdf. 